\title{
Degradation Mechanisms of Platinum Nanoparticle Catalysts in Proton Exchange Membrane Fuel Cells: The Role of Particle Size
}

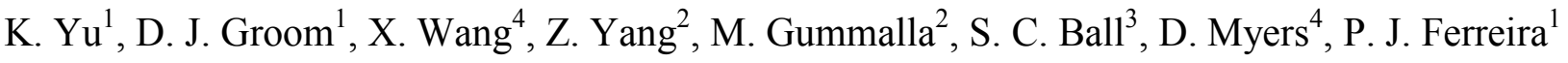 \\ ${ }^{1}$ Materials Science and Engineering Program, The University of Texas at Austin, TX, 78712, USA \\ ${ }^{2}$ United Technology Research Center, CT, USA \\ ${ }^{3}$ Johnson Matthey Technology Center, Blount's Court, Sonning Common, Reading, RG4 9NH, UK \\ ${ }^{4}$ Argonne National Laboratory, Argonne, IL, 60439, USA
}

Proton exchange membrane fuel cells (PEMFCs) are promising energy conversion devices due to their high efficiency, high energy density and low operation conditions. Pt nanoparticles are widely used as the catalysts in cathode and anode for the half cell reactions. However, the durability of Pt nanoparticles still remains the most significant obstacle for large scale application of PEMFCs, especially in the cathode. In general, a significant decrease in electrochemical surface area (ECA) is observed.

In this work, five membrane electrode assemblies (MEAs) with platinum (Pt) nanoparticles of different average sizes $(2.2,3.5,5.0,6.7$, and $11.3 \mathrm{~nm})$ in the cathode were analyzed before and after potential cycling ( 0.6 to $1.0 \mathrm{~V}, 50 \mathrm{mV} / \mathrm{s})$. MEAs with $2.2 \mathrm{~nm}$ and $3.5 \mathrm{~nm}$ show significant growth in mean particle sizes after 10,000 potential cycles, while the other samples do not (Fig.1a).

To understand the aforementioned particle growth, we need to consider the following possible mechanisms: (i) modified electrochemical Ostwald ripening (MEOR), (ii) platinum dissolution and re-precipitation inside the membrane and (iii) particle migration and coalescence. As MEOR is an isotropic process, a comparison of the particle size distributions (PSDs) of spherical particles and PSDs of all the particles indicates that this mechanism plays a significant role in the degradation of $2.2 \mathrm{~nm}$ and $3.5 \mathrm{~nm}$ samples, but not in the other samples (Fig.1b). Re-precipitated particles in the membrane are found among almost all the samples (Figure 2a-e), but their amount is minor comparing to the particles in the cathode, which reveals that re-precipitation plays an insignificant role in the degradation of PEMFCs. In terms of coalescence there are three plausible mechanisms: (i) particles migrate and coalescence, (ii) particles in proximity grow in size due to MEOR and make contact and (iii) soluble Pt species re-precipitate to bridge two particles followed by MEOR (Figure 3a). In any case, coalesced particles occur among all samples, although the $2.2 \mathrm{~nm}$ sample shows the highest extent of coalescence (Fig.3b). However, as the carbon support exhibits a convoluted 3D structure, as shown by in-situ tomography (Fig. 3c,d), it is difficult for particles to coalesce through a migration mechanism.

In summary, Pt dissolution seems to be the controlling mechanism for degradation, as it assists the MEOR process and two plausible mechanisms of coalescence. Thus, reducing Pt dissolution is essential to prevent ECA loss and catalyst performance degradation.

\section{References:}

[1] Z. Yang, S. Ball, D. Condit, M. Gummalla, Journal of The Electrochemical Society, 158, 2011, B1439-B144 

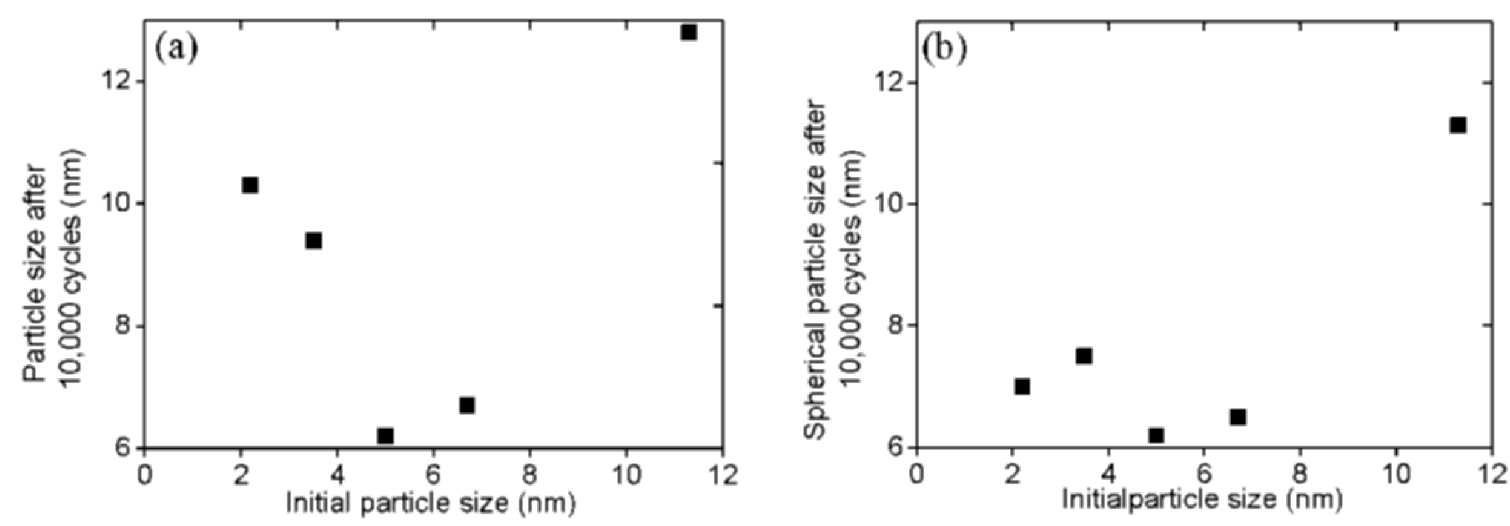

Figure 1. (a) Average particle size after 10,000 cycles for various initial particle sizes. (b) Average spherical particle size after 10,000 cycles for different initial particle sizes.

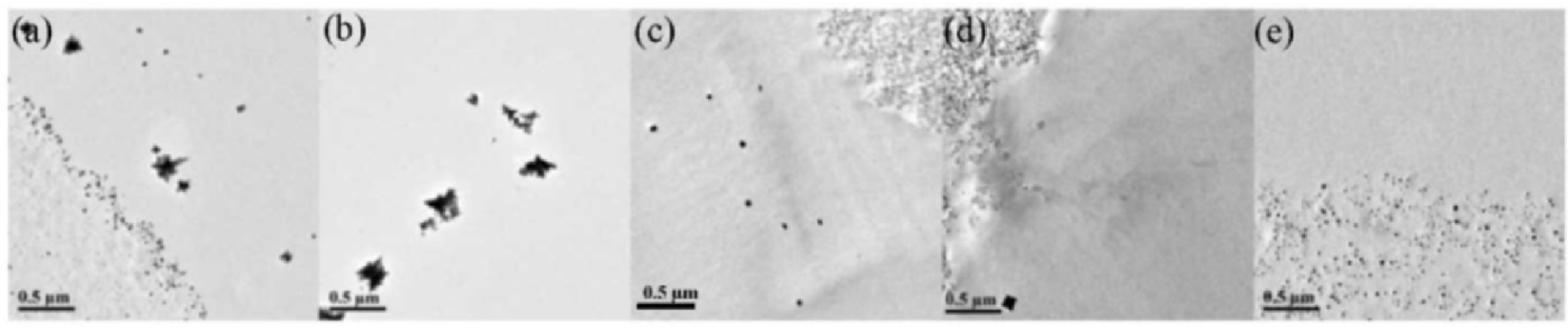

Figure 2. Cathode-membrane interface of MEAs of initial sizes of (a) $2.2 \mathrm{~nm}$, (b) $3.5 \mathrm{~nm}$, (c) $5.0 \mathrm{~nm}$ (d) $6.7 \mathrm{~nm},(\mathrm{e}) 11.3 \mathrm{~nm}$.

(a)

particles migrate and coalesenee

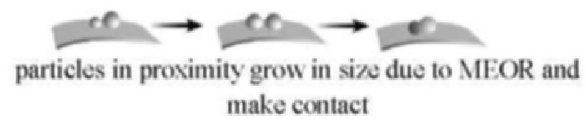

soluble Pt spocios re-precipitate to bridge two particles followud by MEOR

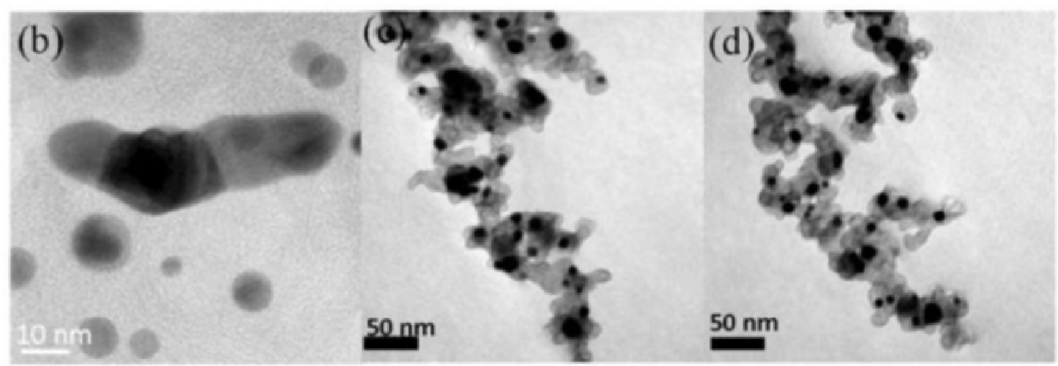

sonm

Figure 3. (a) Coalescence of particle by three plausible mechanisms. (b) Coalesced particles in $2.2 \mathrm{~nm}$ cycled MEA. (c) and (d) In situ tomography images of the carbon support after $-60^{\circ}$ and $+60^{\circ}$ tilts, respectively. 